\title{
Osteomyelitis in an infant with proximal focal femoral deficiency
}

\author{
M Mehtar MBBCh \\ Orthopaedic Registrar \\ L Hartford MBBCh \\ Medical Officer \\ GB Firth MBBCh, FCS(SA)(Orth), MMed(Orth) \\ Orthopaedic Consultant
}

Division of Orthopaedic Surgery, Chris Hani Baragwanath Hospital, University of the Witwatersrand, Johannesburg

\author{
Correspondence: \\ Dr GB Firth \\ Dept. of Orthopaedics \\ Wits Medical School \\ Tel: (011) 717-2538 \\ Fax: (011) 717-2551 \\ Email: greg.firth@gmail.com
}

\begin{abstract}
We present the case of a 6-week-old neonate with proximal focal femoral deficiency (PFFD) complicated by osteomyelitis of the right femur with no preceding trauma or surgical intervention.
\end{abstract}

Key words: osteomyelitis, PFFD, infant, MRI, femur

http:/ / dx.doi.org/10.17159/2309-8309/2016/v15n2a9

\section{Introduction}

Proximal focal femoral deficiency (PFFD) is a rare congenital anomaly characterised by a developmental defect of the proximal part of the femur and hip joint. ${ }^{1,2}$ Clinically the shortened bulky thigh segment is usually held in a flexed, externally rotated and abducted position. ${ }^{1,2}$ PFFD occurs with an incidence of 1 in 52000 live births. ${ }^{3,4}$

This case report highlights the unusual presentation of double pathology of PFFD and associated osteomyelitis of the ipsilateral proximal femur with no preceding trauma or surgical intervention. This case shows how magnetic resonance imaging (MRI) can be useful in localising subperiosteal infections of the proximal femur in the presence of abnormal pre-existing anatomy in very young patients.

\section{Case report}

The mother of the patient, Mrs ZK, a 27-year-old, parity 2 gravidity 3, booked early at approximately 14 weeks of gestation, with normal booking bloods. She had a history of previous Caesarian section for pregnancy-induced hypertension. She presented to Chris Hani Baragwanath Academic Hospital for a foetal anomaly scan at 26 weeks of gestation and was reported to have placenta praevia major and shortening of all the long bones in the right upper and lower limbs. No other structural abnormalities were noted. Three followup scans confirmed these findings. The mother of the patient was admitted from 33 weeks of gestation until delivery. Two doses of betamethasone were administered prior to delivery. The patient was delivered via Caesarean section at 36 weeks of gestation; uncomplicated delivery, baby born with good Apgar scores of 9 at 1 minute and 10 at 5 minutes. 
The birth weight was 2070 grams.

He presented to the Orthopaedic Outpatient Department with a limb length discrepancy and was assessed as having proximal focal femoral deficiency classified as Group B according to the Gillespie and Torode classification and type A according to the Aitken classification. The mother was counselled and follow-up was organised for three months' time.

He however returned to the paediatric ward at six weeks of age with an acute onset of new symptoms. The mother reported that the infant was not moving the right leg and cried with passive movement of the right leg with a few days duration. On examination, the neonate had low grade pyrexia and was irritable, particularly on passive movement of the right hip. He had a pseudo paralysis of the right hip and knee. An assessment of neonatal sepsis was made and the patient was admitted for further investigation and management under the care of the Paediatric Department.

Septic markers on admission were not obviously suggestive of infection with a white cell count of 10.01 and a C-reactive protein of 9.9. The paediatric orthopaedic team was called to see the patient after several days in hospital. Ultrasound of the right hip was done a few days after admission, and showed a shortened right femur but no evidence of septic arthritis of the right hip or any collection of fluid in the proximal femur. An AP pelvis and femur Xray was done after the ultrasound which showed a periosteal reaction around the proximal part of the right femur (Figure 1). The patient was started on intravenous antibiotics (cloxacillin and cefotaxime). The patient had ongoing pyrexia in the days that followed. An MRI scan was ordered and this showed a right femoral pseudarthrosis with a $9 \mathrm{~mm}$ collection posterior to the pseudarthrosis in the proximal part of the femur (Figure 1). Periosteal thickening of the right femur was noted with overlying muscular and subcutaneous oedema.

The patient was taken to theatre 6 days after admission for incision and drainage of the right proximal femur collection. The delay in getting him to theatre occurred because the paediatricians were working him up and treating him as a pyrexia of unknown origin. The diagnosis was not initially clear until the MRI had been done. A postero-lateral approach to the proximal femur was used as directed by MRI findings. Intra-operatively a small amount of pus was found under the periosteum at the level of the pseudarthrosis (Figure 2). Samples were taken for histology and microscopy, and the wound was washed out copiously with normal saline. Histology showed the samples to be consistent with chronic osteomyelitis. Staph. aureus was cultured from the specimen sensitive to cloxacillin. The baby responded to antibiotics and had an uneventful recovery.

At one-year follow-up the baby had a painless hip with full range of motion and no signs of infection clinically or on laboratory investigations. The plain X-ray and repeat MRI scan at one-year follow-up were negative for infection and showed a coxa vara with some development of the right femoral head (Figure 3).
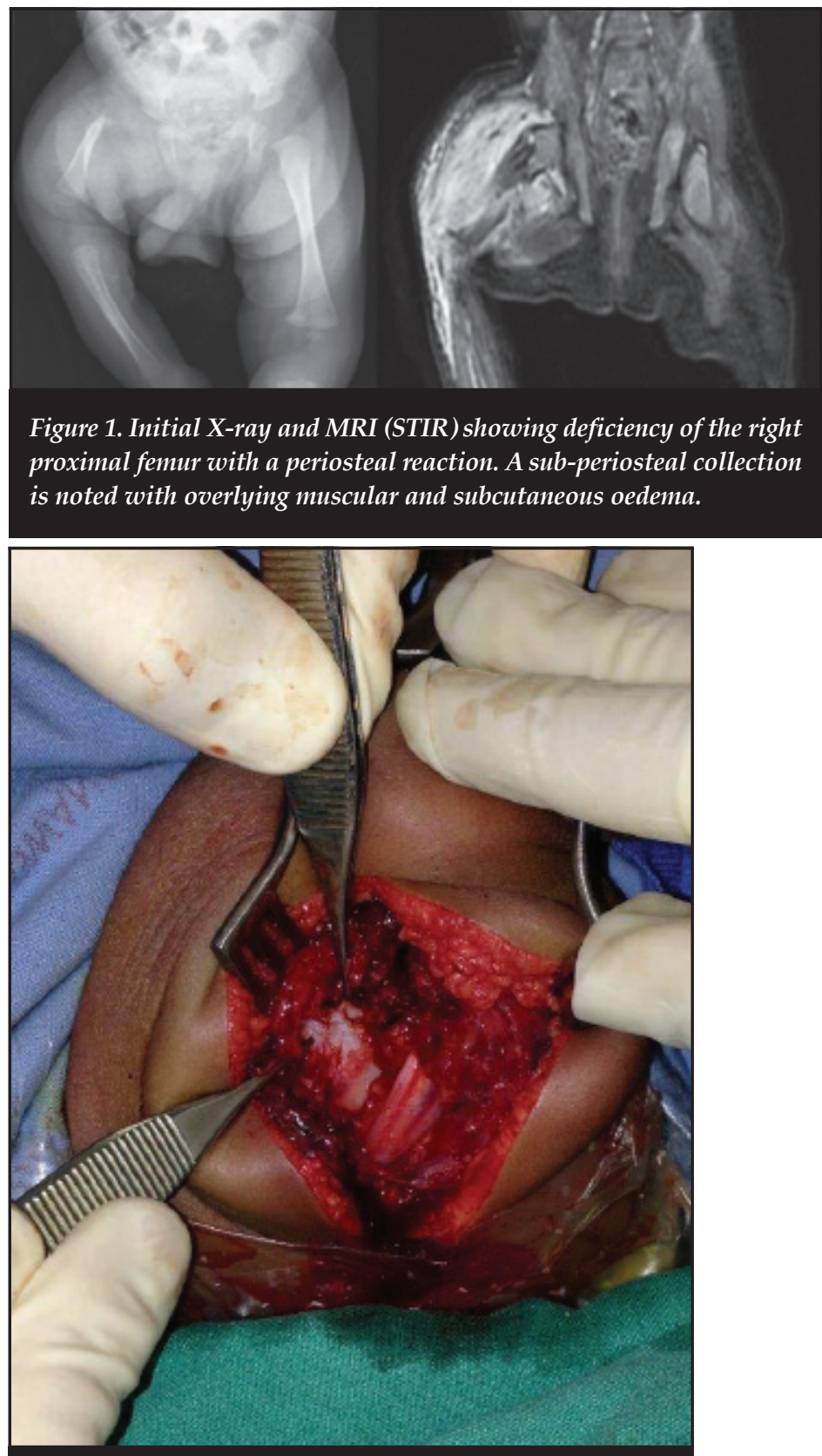

Figure 2. Intra-op picture of right proximal femur demonstrating sub-periosteal femoral collection. The sciatic nerve is shown just posterior to the area of interest.

\section{Discussion}

PFFD is a rare spectrum of proximal femoral deficiencies characterised by failure of normal development of the proximal part of the femur and hip joint. ${ }^{1-3}$ The clinical spectrum of PFFD is wide ranging from minimal shortening (femoral hypoplasia) in mild cases, to complete absence of the proximal femur and acetabulum in the most severe cases. ${ }^{1,3,4}$ Fibular deficiency may be concomitantly present in approximately $50 \%$ of PFFD cases. ${ }^{1,5}$ 


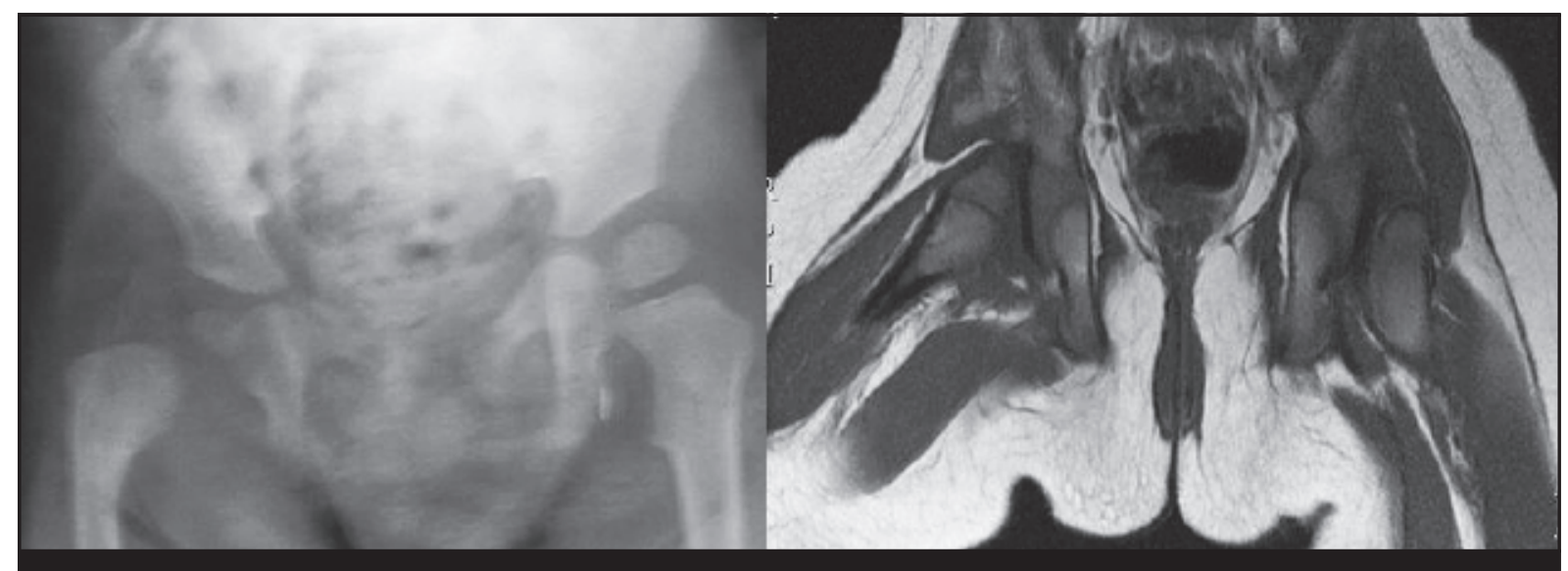

Figure 3. Follow-up X-ray and MRI (T1) at one-year follow-up showing presence of ossific nucleus of the right femoral head which is smaller compared to the left side. No evidence of infection. There is also dysplasia of the right acetabulum. No dissociation seen between the right femoral neck and shaft (Aitken type A).

Clinically patients typically present with a short and bulky thigh that is flexed, externally rotated and abducted. ${ }^{1-3,6} \mathrm{~A}$ clinical paradox exists in that the 'hip joint' in PFFD is generally painless and relatively stable.,8

Westin and Gunderson ${ }^{8}$ noted that 'hip pain is seldom a complaint' and that 'in spite of the complete separation of the shaft from the proximal element, little or no telescoping occurs with weight bearing'. An associated pseudarthrosis of the proximal femur may occur too.

Pirani et al..$^{7}$ postulated that the soft tissues assume a significant role in the stability and weight transfer across the hip in PFFD based on the soft tissue anatomy they observed. The deficiency exists not only in the bony anatomy of the proximal femur, but also the muscle, capsule and soft tissues of the hip joint itself.

Plain X-rays should always be the first investigation used in the diagnosis of PFFD but further investigation is useful in further delineating the extent of the condition. Ultrasound is a valuable imaging modality; however, there are complex bony abnormalities at the junction of the epiphysis and metaphysis that are difficult to appreciate by ultrasound. ${ }^{3,9,10}$ MRI should be considered the modality of choice when assessing PFFD because of its ability to demonstrate the anatomy of the cartilaginous and bony proximal femur, which can facilitate appropriate treatment. ${ }^{3,4,11}$

Osteo-articular infections in neonates are rare and usually present in an atypical way compared to older children. ${ }^{12}$ Wilson et al. ${ }^{12}$ showed that infants can often have a worse outcome compared to older patients in their series possibly due to a delay in diagnosis. Usually the child will present with pseudo paralysis as in the current case. The blood markers are usually less helpful than in the older child - as seen in this case. The most common organism cultured is $S$. aureus but often Gram-negative organisms are cultured in the neonate. ${ }^{13-15}$ Further imaging is usually required to confirm the diagnosis in this age group. ${ }^{13}$ It is also important to differentiate septic arthritis from extra-articular infection. ${ }^{16}$ MRI could provide a precise diagnosis enabling debridement of the epicentre of infection and thus preventing a prolonged infectious process as well as avoiding unnecessary debridement of the joint and joint contamination. ${ }^{16}$

As we suspected an osteitis on the right side clinically, we started the relevant broad spectrum antibiotics (cloxacillin and cefotaxime). Usually our protocol is to drain the collection as soon as possible - in a similar case we would have opened the hip and knee joint - if these were both negative the femur would have been drilled to decompress any intramedullary pus collection. The rationale in this case before surgical drainage was to accurately localise the collection, which required ultrasound, X-ray and MRI, before the abscess could be appropriately identified and drained in such a small patient. There is a good chance that the small subperiosteal abscess, partly treated by the intravenous cloxacillin given by the paediatricians, would never have been localised without the MRI, potentially causing a chronic osteomyelitis of the femur in this atypical case.

The presence of the PFFD in this case complicated things and so it was decided to investigate further with imaging studies. The X-ray did show a periosteal reaction of the proximal femur but was unable to isolate the exact site of the infection. A technetium bone scan could also have been done to localise the infection, but after discussion with the paediatricians, the MRI was done instead. The ultrasound did not locate the collection which could only be seen on the MRI. The MRI was extremely useful in identifying the exact position of the collection for pre-operative planning. In this atypical case of PFFD and femoral osteomyelitis, further investigation was directed by the change in the clinical findings of the child. Ultrasound findings were equivocal but MRI assisted in identifying the source of infection and enabled appropriate surgical intervention. 


\section{Conclusion}

This atypical case highlights the importance and superiority of MRI in assessing osteomyelitis in a neonate, in the setting of a second pathology - in this case PFFD. The appropriate accurate surgical drainage was performed with the help of the MRI which facilitated a favourable outcome.

\section{Compliance with Ethics Guidelines}

M Mehtar, L Hartford and GB Firth declare they have no conflict of interest.

\section{References}

1. Westberry DE, Davids JR. Proximal focal femoral deficiency (PFFD): management options and controversies. Hip Int 2009;19:S18-25.

2. Koman LA, Meyer LC, Warren FH. Proximal femoral focal deficiency: natural history and treatment. Clin Orthop Relat Res 1982;162:135-43.

3. Biko DM, Davidson R, Pena A, Jaramillo D. Proximal focal femoral deficiency: evaluation by MR imaging. Pediatr Radiol 2012;42:50-56.

4. Dillon JE, Connolly SA, Connolly LP. MR imaging of congenital/developmental and acquired disorders of the pediatric hip and pelvis. Magn Reson Imaging Clin N Am 2005;13:783-97.

5. Koman LA, Meyer LC, Warren FH. Proximal femoral focal deficiency: a 50 year experience. Dev Med Child Neurol 1982;24:344-55.

6. Epps $\mathrm{CH}$. Current concepts review proximal femoral focal deficiency. J Bone Joint Surg Am 1983;65:867-70.

7. Pirani S, Beauchamp RD, Li D, Swatzky B. Soft tissue anatomy of proximal femoral focal deficiency. J Pediatr Orthop 1991;11:563-70.
8. Westin GW, Gunderson FO. Proximal focal femoral deficiency: a review of treatment experiences. In: Aitken GT (eds). Proximal focal femoral deficiency: a congenital anomaly. Washington, D.C.: National Academy of Sciences; 1969: 100-10.

9. Grisson LE, Harcke T. Sonography in congenital deficiency of the femur. J Pediatr Orthop 1994;14:29-33.

10. Kayser R, Mahlfeld K, Grasshoff H, Merk HR. Proximal focal femoral deficiency-a rare entity in the sonographic differential diagnosis of developmental dysplasia of the hip. Ultraschall in Med 2005;26:379-84.

11. Maldjian C, Patel TY, Klein RM, Smith RC. Efficacy of MRI in classifying proximal focal femoral deficiency. Skeletal Radiol 2007;36:215-20.

12. Wilson NIL, Di Paola M. Acute septic arthritis in infancy and childhood. 10 years' experience. J Bone Joint Surg Br 1986;68-B:584-87.

13. Goergens ED, McEvoy A, Watson M, Barrett IR. Acute osteomyelitis and septic arthritis in children. J Paediatr Child Health 2005;41:59-62.

14. Lavy CBD, Thyoka M, Pitani AD. Clinical features and microbiology in 204 cases of septic arthritis in Malawian children. J Bone Joint Surg Br 2005;87-B:1545-48.

15. Robertson AJF, Firth GB, Truda C, Ramdass DA, Groome M, Madhi S. Epidemiology of acute osteoarticular sepsis in a setting with a high prevalence of pediatric HIV infection. J Pediatr Orthop 2012;32:215-19.

16. Mignemi ME, Menge TJ, Cole HA, Mencio GA, Martus JE, Lovejoy S, et al. Epidemiology, diagnosis, and treatment of pericapsular pyomyositis of the hip in children. J Pediatr Orthop 2014;34:316-25.

This article is also available online on the SAOA website (www.saoa.org.za) and the SciELO website (www.scielo.org.za). Follow the directions on the Contents page of this journal to access it. 